\title{
COAGULAÇÃO INTRAVASCULAR DISSEMINADA
}

\author{
DISSEMINATED INTRAVASCULAR COAGULATION
}

Maria Carolina Tostes Pintão' \& Rendrik F. Franco²

\begin{abstract}
'Pós-Graduanda (Hematologia) do Departamento de Clínica Médica da FMRP-USP.
${ }^{2}$ Professor Livre-Docente de Hematologia e Hemoterapia. Coordenador do Serviço de Investigação em Hemofilia e Trombofilia, Fundação Hemocentro de Ribeirão Preto, Coordenador do Laboratório de Hemostasia, Hospital das Clínicas da Faculdade de Medicina de Ribeirão Preto, Universidade de São Paulo.

Correspondência: Dra. Maria Carolina Tostes Pintão - Laboratório de Hemostasia - Hospital das Clínicas da Faculdade de Medicina de Ribeirão Preto - Universidade de São Paulo. Tel. 166022223 - Fax: 166331144 - E-mail: cpintao@hotmail.com
\end{abstract}

PINTÃO MCT \& FRANCO RF. Coagulação intravascular disseminada. Medicina, Ribeirão Preto, 34: 282-291, jul./dez. 2001.

RESUMO: No presente artigo, revisamos diversos aspectos da etiologia, fisiopatologia, diagnóstico e tratamento da coagulação intravascular disseminada (CIVD).

UNITERMOS: Coagulação Intravascular Disseminada. Coagulação. Inflamação.

\section{INTRODUÇÃO}

A coagulação intravascular disseminada (CIVD) é, atualmente, definida como uma síndrome adquirida, caracterizada pela ativação difusa da coagulação intravascular, levando à formação e deposição de fibrina na microvasculatura ${ }^{(1)}$. O sistema fibrinolítico está, em geral, amplamente inativado durante a fase de maior ativação da coagulação, o que contribui para a deposição de fibrina. Porém, em algumas situações (por exemplo, na leucemia promielocítica aguda) a fibrinólise pode estar acelerada, contribuindo, assim, para quadros de sangramento grave. A deposição de fibrina pode levar à oclusão dos vasos e conseqüente comprometimento da irrigação sangüínea de diversos órgãos, o que, em conjunto com alterações metabólicas e hemodinâmicas, contribui para a falência de múltiplos órgãos. O consumo e conseqüente depleção dos fatores da coagulação e plaquetas, resultantes da contínua atividade procoagulante, pode levar a sangramento difuso, o que freqüentemente é a primeira manifestação notada. É importante ressaltar que a CIVD é sempre secundária a uma doença de base e a identificação e trata- mento da condição predisponente são fundamentais para a resolução da síndrome.

Apesar de ter sido extensivamente estudada nas últimas décadas, muitos aspectos da CIVD, particularmente aqueles relacionados à sua definição, diagnóstico e tratamento, são ainda motivo de extensos debates. Este capítulo apresenta informações atualizadas relativas à fisiopatologia, aspectos clínicos, diagnóstico e tratamento da CIVD.

\section{CONDIÇÕES CLÍNICAS ASSOCIADAS À CIVD}

A CIVD pode ocorrer em associação a uma grande variedade de condições clínicas, as principais listadas na Figura 1. A incidência da síndrome nas diferentes situações citadas não pode ser estabelecida com precisão, em parte pela dificuldade em defini-la e também pela falta de critérios diagnósticos claros $^{(1,2)}$. As principais condições clínicas envolvidas na etiologia da CIVD serão discutidas a seguir.

As doenças infecciosas, em particular a septicemia, são as mais comumente associadas à CIVD. 
Apesar de virtualmente qualquer microorganismo (como vírus e parasitas) desencadear a síndrome, as infecções bacterianas são as mais freqüentemente associadas à CIVD. Cerca de $30 \%$ a $50 \%$ dos pacientes em septicemia cursam com CIVD em fase II ou III e, virtualmente, todos apresentam CIVD em fase I (Figura 6). Diferente do que se acreditava, a ocorrência de CIVD em septicemia por germes do tipo gram-positivo é tão comum quanto na septicemia por gram-negativo. Diversos fatores, como componentes de membrana dos microorganismos (lipopolissacarídeos ou endotoxinas) e exotoxinas de bactérias (por exemplo, $\alpha$-toxina estafilocócica), estão envolvidos e resultam em resposta inflamatória generalizada, com liberação sistêmica de citocinas que estão diretamente envolvidas no distúrbio hemostático que caracteriza o quadro de $\operatorname{CIVD}^{(1,2,3)}$.

Trauma grave também é freqüentemente relacionado à CIVD e uma combinação de mecanismos, que inclui liberação de gordura e fosfolipídios tissulares na circulação, hemólise e lesão endotelial, contribui para a ativação sistêmica da coagulação em tal situação. O padrão de liberação de citocinas em pacientes politraumatizados é semelhante àquele observado em pacientes sépticos, reforçando as evidências do seu papel fundamental no desenvolvimento da síndrome. A incidência de CIVD em pacientes que sofreram trauma grave com síndrome da resposta inflamatória sistêmica é de $50 \%$ a $70 \%{ }^{(1,2)}$.

Tumores sólidos e neoplasias hematológicas podem cursar com CIVD. O mecanismo envolvido é ainda pouco compreendido, mas parece estar relacionado ao fator tissular (FT), expresso na superfície das células tumorais. Dez a 15\% dos pacientes com tumores metastáticos e $15 \%$ a $20 \%$ dos pacientes com leucemia exibem evidências de ativação intravascular da coagulação. A leucemia promielocítica aguda cursa com uma forma distinta de CIVD, caracterizada por hiperfibrinólise e, apesar de o sangramento ser a manifestação clínica mais comum nesses pacientes, a trombose disseminada também ocorre ${ }^{(1,2)}$.

A CIVD é uma complicação clássica de condições obstétricas, como descolamento de placenta e embolia de líquido amniótico. A liberação de material tromboplástico é o que provavelmente desencadeia a síndrome, uma vez que o grau de descolamento placentário correlaciona-se com a gravidade do quadro e o líquido amniótico é um potente ativador da coagulação in vitro. A pré-eclâmpsia e eclâmpsia também podem cursar com CIVD, no entanto, a coagulopatia que faz parte da síndrome HELLP (hemolysis, elevated liver enzymes and low platelets) caracteriza-se melhor como secundária à anemia hemolítica microangiopática, situação distinta da CIVD e discutida abaixo ${ }^{(1,2)}$. Outras causas obstétricas de CIVD encontram-se listadas na Figura 1.

Doenças vasculares, como hemangiomas gigantes (síndrome de Kasabach-Merritt) ou grandes aneurismas de aorta, podem resultar em ativação local da coagulação e ter como consequiência a depleção sistêmica dos fatores da coagulação e plaquetas, consumidos localmente. Com menor freqüência, fatores ativados podem atingir a circulação sistêmica, desencadeando a CIVD. A incidência de CIVD clinicamente detectável em pacientes com hemangioma gigante é de $25 \%$ e em aneurisma de aorta é de $0,5 \%$ a $1 \%(1,2)$.

Vale ainda mencionar um grupo de doenças caracterizado por anemia hemolítica microangiopática e que inclui púrpura trombocitopênica trombótica (PTT), síndrome hemolítico-urêmica (SHU), anemia hemolítica microangiopática induzida por quimioterapia, hipertensão maligna e síndrome HELLP. O evento comum nessas doenças é a lesão endotelial, que causa adesão e agregação plaquetária, formação de

\section{Medicina Interna}

Sepsis/infecções, leucemia, transplante, insuficiência hepática aguda, reações alérgicas, acidente ofídico, hipotermia, deficiência homozigotica de PC, tumores sólidos, cirrose, vasculite, Síndrome Kasabach-Merritt, SARA

\section{Cirurgia}

Trauma, grandes operações, lesão SNC, embolia gordurosa, queimaduras, cirurgia cardíaca bypass, transplante de órgãos, aneurisma aorta, tumores vasculares

\section{Obstetrícia}

Embolia de líquido amniótico, DPP, aborto séptico, rutura uterina, DHEG, sepsis, HELLP, feto morto retido

\section{Medicina transfusional}

Reação hemolítica transfusional aguda, transfusão maciça

Figura 1: Condições clínicas associadas à CIVD. 
trombo e alteração da fibrinólise. A patogênese da PTT e da SHU envolve a deficiência de uma protease que cliva multímeros de fator de von Willebrand, levando ao seu acúmulo anormal e hiperagregação plaquetária. A coagulopatia presente em tais doenças envolve, essencialmente, o consumo de plaquetas e não dos fatores da coagulação e é, portanto, distinta daquela relacionada $\operatorname{CIVD}^{(1,2)}$.

\section{FISIOPATOLOGIA}

Nos últimos anos, estudos envolvendo pacientes sépticos e modelos animais e humanos de endotoxemia e septicemia contribuíram em muito para a compreensão da fisiopatologia da CIVD. Em linhas gerais, pode-se dizer que a deposição sistêmica de fibrina é resultado da geração de trombina, mediada pelo complexo fator tissular/fator VII ativado (FT/ FVIIa) e da inibição ou disfunção dos anticoagulantes naturais (antitrombina [AT], proteína C [PC], proteína $\mathrm{S}$ [PS] e inibidor da via do fator tissular [TFPI]). Em adição, a inibição da atividade fibrinolítica pelo aumento dos níveis do inibidor do ativador do plasminogênio do tipo 1 (PAI-1) resulta em remoção inadequada de fibrina, contribuindo, dessa forma, para a trombose da microvasculatura. Citocinas, principalmente a interleucina-6, têm ação central nesse processo (Figura 2). A ativação sistêmica da coagulação promove não somente deposição de fibrina e trombose, mas também, consumo e conseqüente depleção dos fatores da coagulação e plaquetas, o que, freqüentemente, resulta em manifestações hemorrágicas. No conjunto, esses mecanismos fisiopatológicos explicam a ocorrência simultânea de trombose e sangramento na CIVD (Figura 3). Nesse ponto, deve ser salientado que, não obstante as manifestações hemorrágicas sejam freqüentemente observadas ao exame clínico, é a trombose microvascular que, provavelmente, mais contribui para a disfunção de órgãos-alvo e mortalidade associadas à CIVD.

Geração de trombina. Diversas evidências apontam para o complexo FT/FVIIa como principal mediador da geração de trombina. O bloqueio da via do FT/FVIIa por anticorpos monoclonais anti-FT ou anti-FVIIa resulta em completa inibição da geração de trombina e previne a ocorrência de CIVD e morte em primatas submetidos a infusão de endotoxina ${ }^{(1,2,4)}$. Adicionalmente, em estudo recente, foi demonstrado que a expressão de FT in vivo encontra-se aumentada em modelo de endotoxemia humana, e é diretamente associada à marcante ativação da coagulação ${ }^{(5)}$. Experimentos envolvendo o sistema de contato da coagulação (previamente designado "via intrínseca") mostraram que tal via não contribui de modo importante para a geração de trombina, mas exerce funções relacionadas a outros sintomas da resposta inflamatória sistêmica como, por exemplo, a ocorrência de hipotensão ${ }^{(1,2,4)}$.

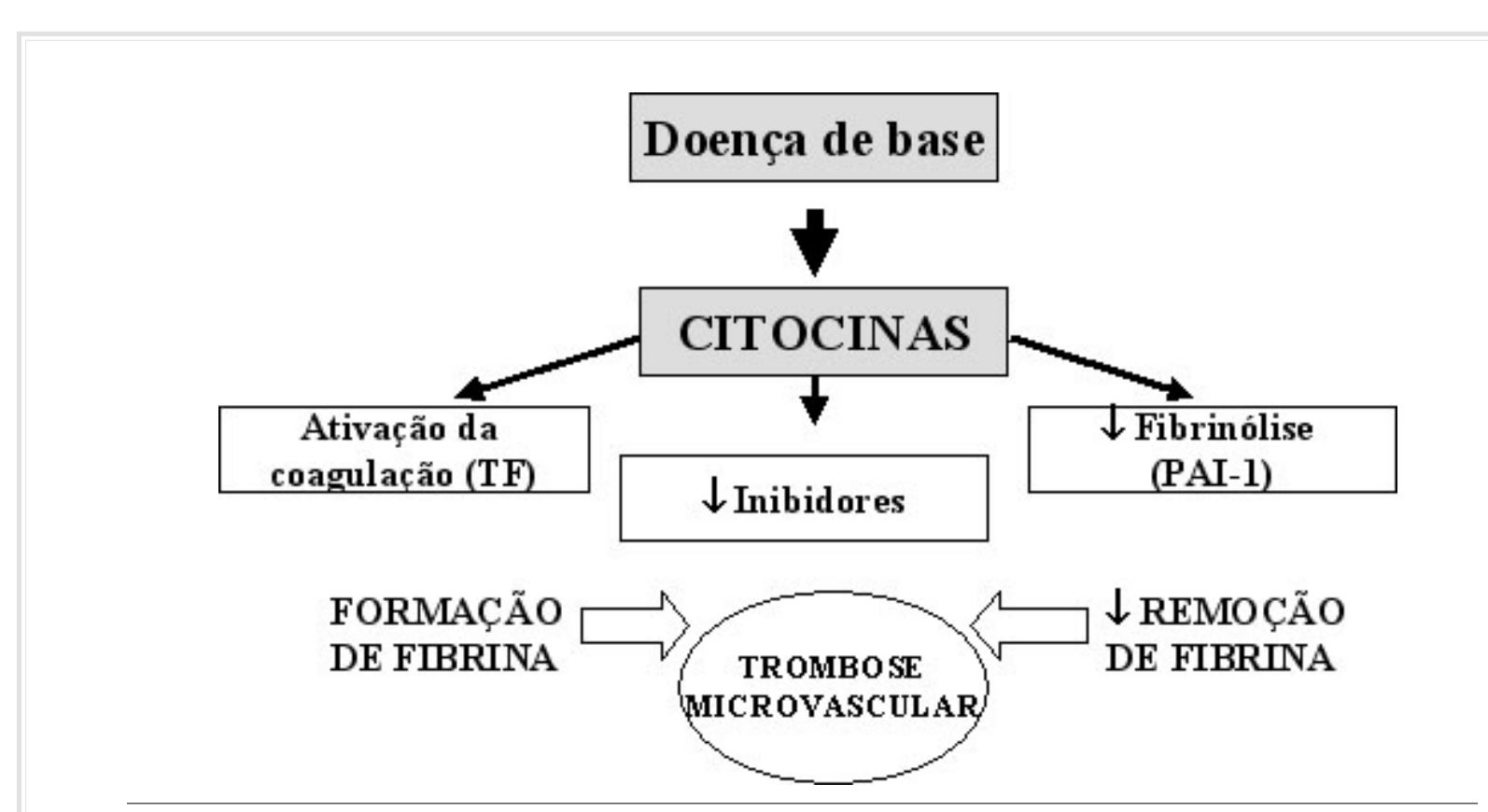

Figura 2: Patogênese da CIVD 

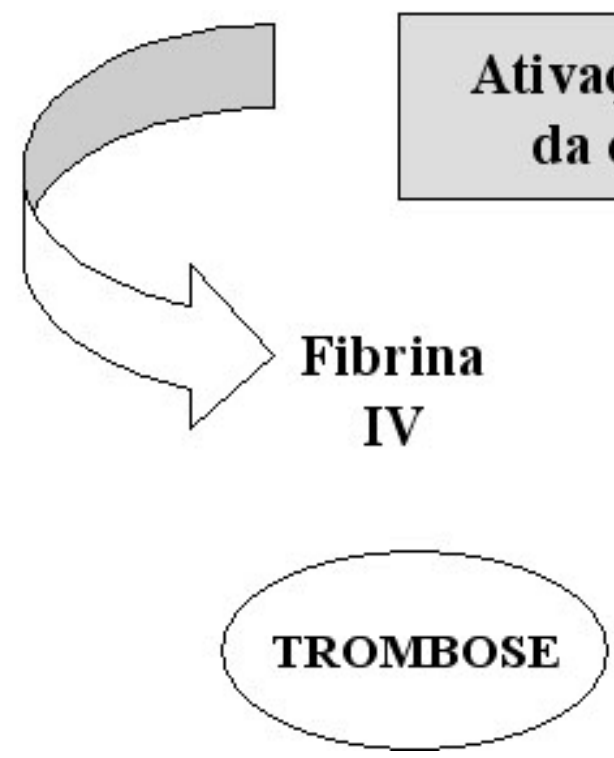

Ativação sistêmica da coagulação

Figura 3: Mecanismos fisiopatológicos na CIVD.

Supressão dos inibidores fisiológicos da coagulação. A atividade dos principais reguladores da ativação da coagulação (AT, PC, PS e TFPI) está inibida nos pacientes com CIVD, contribuindo para a formação e deposição de fibrina na microvasculatura. Os níveis plasmáticos de AT estão reduzidos, em conseqüência do consumo secundário à geração contínua de trombina, aumento da degradação pela elastase liberada por neutrófilos ativados e diminuição da sua síntese. A atividade do sistema PC/PS também se encontra diminuída em decorrência de consumo e da diminuição da expressão de trombomodulina nas células endoteliais, além da diminuição da fração livre da PS, importante cofator da atividade da PC. Os níveis plasmáticos de TFPI, em geral, não estão reduzidos, porém há evidências de que sua atividade reguladora não seja exercida adequadamente em pacientes com $\operatorname{CIVD}^{(1,2,4)}$.

Fibrinólise. Modelos experimentais demonstraram que a bacteremia e a endotoxemia resultam num rápido aumento da atividade fibrinolítica seguida de sua supressão, sustentada pelo aumento mantido nos níveis plasmáticos do PAI-1, o principal inibidor do sistema fibrinolítico. Com efeito, sua atividade remanescente não é capaz de compensar a deposição sistêmica maciça de fibrina (Figura 2). Exceção se faz à coagulopatia relacionada à leucemia promielocítica aguda, em que evidências de fibrinólise intensa estão usualmente presentes ${ }^{(1,2,4)}$.
Inflamação e coagulação. A relação existente entre inflamação e coagulação é inquestionável. A reação inflamatória ativa a coagulação sangüínea por promover a expressão de fator tissular no espaço intravascular, eliciar a expressão de moléculas de adesão de leucócitos na parede vascular, diminuir a atividade fibrinolítica e a função da via anticoagulante da $\mathrm{PC}^{(6)}$. As citocinas são importantes mediadores dessas alterações e seu papel no desenvolvimento da CIVD vem se tornando cada vez mais claro. A síndrome da resposta inflamatória sistêmica, secundária a infecções e lesões teciduais extensas, é mediada por diversas citocinas capazes de ativar a coagulação in vitro. Diferentes investigações demonstraram que o fator de necrose tumoral- $\alpha$ (TNF- $\alpha$ ) é o primeiro a se elevar, seguido da elevação dos níveis plasmáticos de interleucina 6 (IL-6) e interleucina 1 (IL-1). Estudos envolvendo a administração dessas interleucinas em voluntários saudáveis ou em modelos animais bem como o uso de anticorpos bloqueando sua ação, sugeriram que a IL-6 é a principal responsável pela geração de trombina, possivelmente por regular a expressão de fator tissular. O papel do TNF- $\alpha$ na ativação da coagulação é relacionado à sua capacidade de induzir o aumento da IL-6. Adicionalmente, o TNF- $\alpha$ parece ser o principal mediador da depressão da atividade do sistema da PC, por induzir diminuição da expressão de trombomodulina nas células endoteliais (Figura 4). A IL-1 é um potente agonista da expressão 


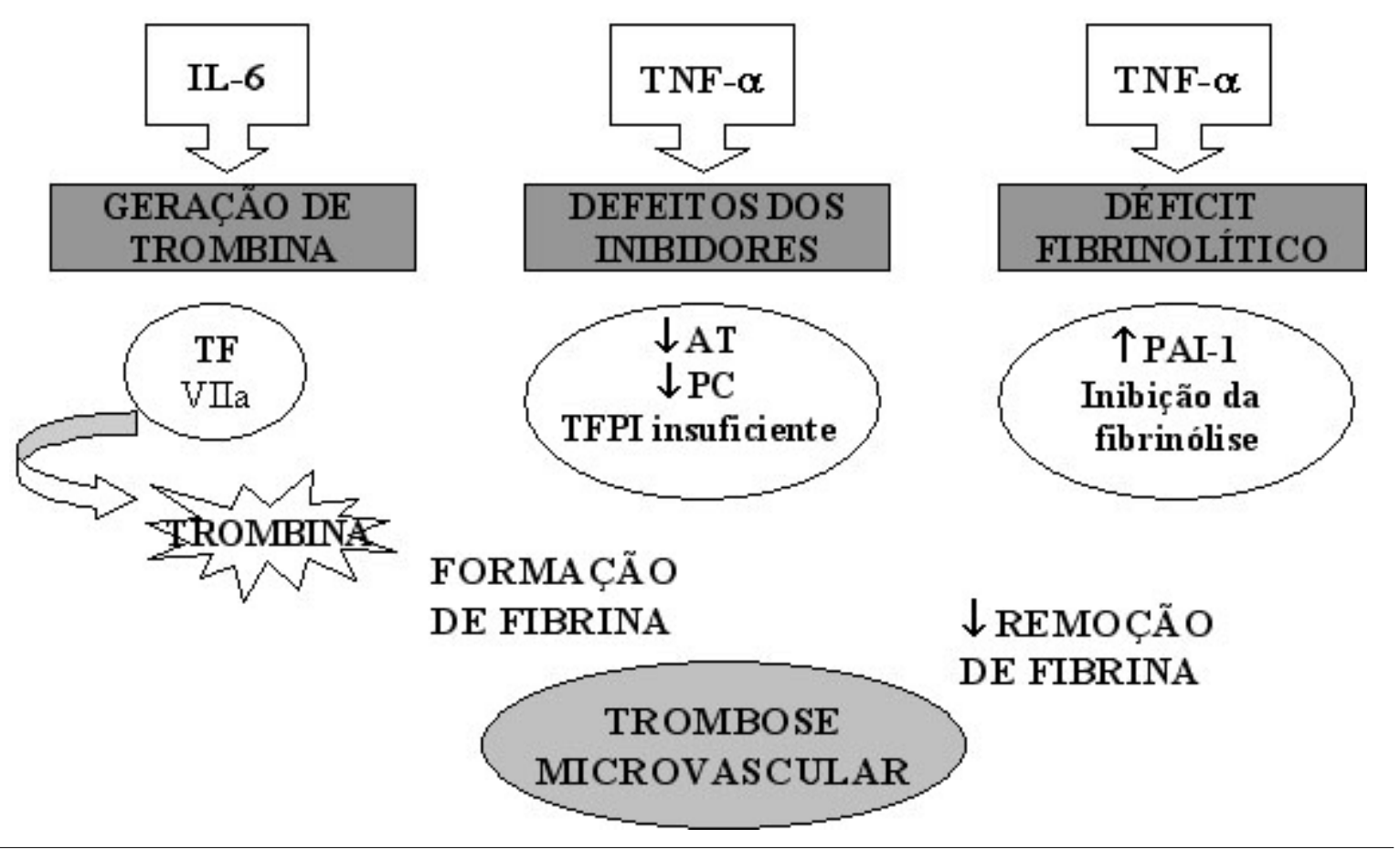

Figura 4: Papel das interleucinas na CIVD.

de fator tissular in vitro, no entanto, seu papel in vivo ainda é pouco claro e o fato de sua elevação só ocorrer depois das principais alterações procoagulantes que seguem a administração de endotoxinas, torna a possibilidade de um papel direto no desenvolvimento da CIVD pouco provável ${ }^{(2,7,8)}$.

Por outro lado, a ativação descontrolada do sistema de coagulação não resulta apenas em trombose, mas também em inflamação e proliferação celular, mediadas principalmente pela ação da trombina. Assim, estabelece-se uma alça de retroalimentação positiva que, se não controlada, pode progredir, promovendo lesão vascular, falência de múltiplos órgãos e morte. Os mecanismos controladores desse processo ainda não foram completamente elucidados, porém a PC parece ser um importante mediador do controle, o que pode, ao menos em parte, explicar os resultados promissores obtidos com o uso desse inibidor fisiológico da coagulação no tratamento de pacientes sépticos graves ${ }^{(6,9)}$.

O significado clínico da presença da CIVD não é completamente claro. É indiscutível a importância da plaquetopenia e depleção de fatores da coagulação em pacientes com sangramento ou submetidos a procedimentos invasivos. Entretanto, a magnitude da contribuição da deposição de fibrina na microvascula- tura para a falência de múltiplos órgãos e morte é ainda desconhecida. De qualquer forma, diversas evidências apontam para a contribuição da CIVD em tal processo e uma hipótese atraente é a de que as interações entre coagulação e inflamação, como expostas acima, estejam diretamente envolvidas ${ }^{(10,11)}$.

\section{DIAGNÓSTICO}

Não existe exame laboratorial que isoladamente estabeleça ou afaste o diagnóstico de CIVD, porém a combinação de alterações clínicas e laboratoriais compatíveis e, principalmente, a presença de doença sabidamente relacionada à síndrome permitem diagnóstico confiável na maioria dos $\operatorname{casos}^{(1,2)}$.

Clinicamente, pode-se observar os sinais de resposta inflamatória sistêmica, como febre, hipotensão, acidose, manifestações de sangramento difuso (petéquias, equimoses, sangramento em locais de punção venosa e cicatriz cirúrgica ou traumática) e sinais de trombose ${ }^{(12)}$. As principais manifestações clínicas são apresentadas na Figura 5. A CIVD é um processo de gravidade progressiva, o que permite caracterizar sua evolução em fases, conforme mostrado na Figura $6^{(13)}$. 


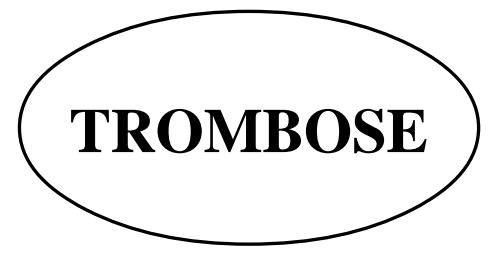

SN: $\downarrow$ consc., delírio, coma

Pele: isquemia focal, gangrena

Rins: oligúria, azotemia

AR: SARA

GI: ulceração aguda

Anemia hemolítica

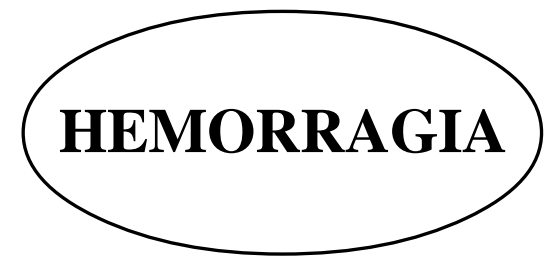

SN: sangramento i.c.

Pele: petéquias, equimoses, sítios de venopunção

Mucosas: epistaxis, gengivorragia

Rins: hematúria

GI: sangramento

Figura 5: CIVD: quadro clínico

Fase 1 (ativação compensada)

- Poucos sintomas

- TTPA, TP, TT, fibrinogênio: $\mathrm{N}$

- Plaquetas: N/limite

- AT: $\downarrow$ discreta

- DD, PDF, TAT, F1+2: $\uparrow$

Fase 2 (ativação descompensada)

- Sangramentos + disfunção de órgãos

- TTPA, TP, TT: $\uparrow$

- Plaquetas, fibrinogênio: $\downarrow$

- AT, fatores da coagulação: $\downarrow$

- DD, PDF, TAT, F1+2: $\uparrow \uparrow$

Fase 3 (CIVD plenamente manifesta)

- Sangramentos + disfunção de múltiplos órgãos

- TTPA, TP, TT: $\uparrow \uparrow / \uparrow \uparrow \uparrow$

- Plaquetas, AT, fibrinogênio, fatores: $\downarrow \downarrow$

- DD, PDF, TAT, F1+2: $\uparrow \uparrow \uparrow$

Figura 6: Fases da CIVD: manifestações clínicas e laboratoriais
Os exames laboratoriais necessários para a confirmação diagnóstica de CIVD são discutidos a seguir.

Tempo de protrombina (TP), tempo de tromboplastina parcial ativada (TTPa) e tempo de trombina (TT). O prolongamento do TP e do TTPa reflete o consumo dos fatores da coagulação e, portanto, uma fase de ativação franca da coagulação. O resultado normal desses exames não exclui a presença de CIVD, uma vez que, nas fases iniciais da síndrome, não há consumo suficiente de fatores da coagulação para prolongar o TP e o TTPa. Eles são exames amplamente disponíveis e sua realização seriada, diante da suspeita de CIVD, permite avaliar a evolução do quadro assim como a resposta terapêutica. O TT, por sua vez, reflete a hipofibrinogenemia relacionada ao consumo de fibrinogênio, além de alterar-se mediante ação dos produtos de degradação da fibrina/ fibrinogênio (PDFs) sobre o fibrinogênio.

Contagem de plaquetas. A contagem de plaquetas inicialmente baixa e, particularmente, a sua queda progressiva são um achado sensível, ainda que pouco específico, para avaliar a evolução da síndrome, uma vez que a agregação plaquetária é consequiência da geração de trombina. A estabilização da contagem de plaquetas sugere que a formação de trombina tenha cessado.

Fibrinogênio e produtos da degradação da fibrina (PDFs). A dosagem de fibrinogênio plasmático 
deve ser feita, porém em fases iniciais da CIVD, seus níveis podem permanecer normais ou mesmo elevados apesar da ativação da coagulação, uma vez que se trata de proteína de fase aguda. A hipofibrinogenemia aparece em casos graves de CIVD. O aumento dos PDFs são, em geral, observados desde o início do quadro de CIVD. Nos últimos anos mostrou-se que testes de quantificação de dímeros-D (um dos produtos da degradação da fibrina no plasma) são mais sensíveis que os ensaios de PDFs e que níveis normais de dímeros-D têm um alto valor preditivo negativo para a presença de degradação intravascular da fibrina. Uma vez que o fibrinogênio é também degradado em regiões extravasculares, a elevação dos PDFs e dos dímeros-D não implica necessariamente a presença de fibrinólise intravascular. Deve-se ainda considerar que PDFs são metabolizados pelo fígado e excretados pelos rins e, portanto, os níveis plasmáticos desses produtos são influenciados pela função dos órgãos citados.

Dosagem de fatores da coagulação e anticoagulantes naturais (proteína $C$, antitrombina). A diminuição dos níveis plasmáticos de fatores da coagulação é refletida, como já foi dito, no prolongamento dos tempos de coagulação e é resultado do seu consumo. A dosagem de fatores específicos (por exemplo, fatores V e VIII) pode ser útil em algumas situações, como, para auxiliar na diferenciação entre coagulopatia associada à insuficiência hepática e CIVD. A dosagem plasmática de AT e PC pode também ter valor diagnóstico complementar em casos em que a hipótese de CIVD não pôde ser estabelecida mesmo após a realização dos exames mencionados anteriormente.

Marcadores de ativação da coagulação: fibrinopeptídeo A (FPA), fragmento $_{1+2}$ da protrombina $\left(F_{1+2}\right)$, fibrina solúvel e complexo trombinaantitrombina (TAT). Considerando-se a definição de CIVD (que leva em conta a formação intravascular de fibrina), a medida direta dos níveis de fibrina solúvel no plasma poderia contribuir para o diagnóstico definitivo da síndrome. No entanto, testes dessa natureza não se encontram disponíveis ou validados para utilização na rotina de investigação diagnóstica da CIVD. Os marcadores FPA, $\mathrm{F}_{1+2}$ e TAT são indicadores sensíveis da geração de trombina, com sensibilidade e especificidade que variam de 80 a $90 \%$, porém são exames caros e, em geral, não disponíveis para uso rotineiro. Apesar de úteis em situações clínicas de maior complexidade, não são essenciais na prática clínica diária.
Observação do esfregaço de sangue periférico. Tem importância nos casos de suspeita de CIVD, à medida que a presença de hemácias fragmentadas (esquisócitos) é indicativa da presença de trombose microvascular.

Em suma, diante da suspeita de CIVD, os seguintes exames devem ser realizados: contagem de plaquetas e visualização do esfregaço de sangue periférico, TP, TTPa, TT, dosagem de fibrinogênio, PDFs e dímeros-D. É importante enfatizar que a repetição seriada desses exames tem maior valor que seus resultados isoladamente e permite melhor avaliação da evolução da síndrome. Em casos selecionados, a depender de análise individual e disponibilidade do método diagnóstico, dosagens de AT, PC ou fatores da coagulação podem também ser efetuadas.

Scores. Nas últimas décadas, numerosos sistemas de score foram propostos com a intenção de permitir diagnóstico e acompanhamento mais precisos da CIVD, porém, até o momento, nenhum deles foi amplamente aceito. Recentemente, o Subcomitê Científico de CIVD da Sociedade Internacional de Trombose e Hemostasia (ISTH) propôs um sistema que considera duas situações diferentes: CIVD fases I/II e CIVD plenamente manifesta ${ }^{(14)}$. O diagnóstico de CIVD plenamente manifesta é baseado na aplicação de algoritmo de cinco passos, com atribuição de pontos cuja soma maior ou igual a cinco é compatível com o diagnóstico de CIVD plenamente manifesta, levando-se em conta que uma pontuação menor não exclui o diagnóstico (Figura 7). A presença de uma doença de base sabidamente associada à síndrome, é o primeiro passo do algoritmo e condição sine qua non para sua execução. $\mathrm{O}$ subcomitê recomenda que o score seja calculado diariamente a fim de que se caracterize a gravidade e a evolução do quadro ${ }^{(14)}$.

\section{TRATAMENTO}

Evidências seguras que possam fundamentar o tratamento da CIVD ainda são escassas, e a maioria dos tópicos envolvidos em tal tratamento é motivo de controvérsia. No entanto, é de geral aceitação que a pedra angular do manejo da CIVD é o tratamento da doença de base. Enquanto isto se faz, estratégias de "suporte" (administração de fluidos, antibioticoterapia, correção de distúrbios hidroeletrolíticos e do equilíbrio ácidobásico, suporte ventilatório e cardiocirculatório) podem ser necessárias. Adicionalmente, medidas baseadas principalmente nos conhecimentos que 


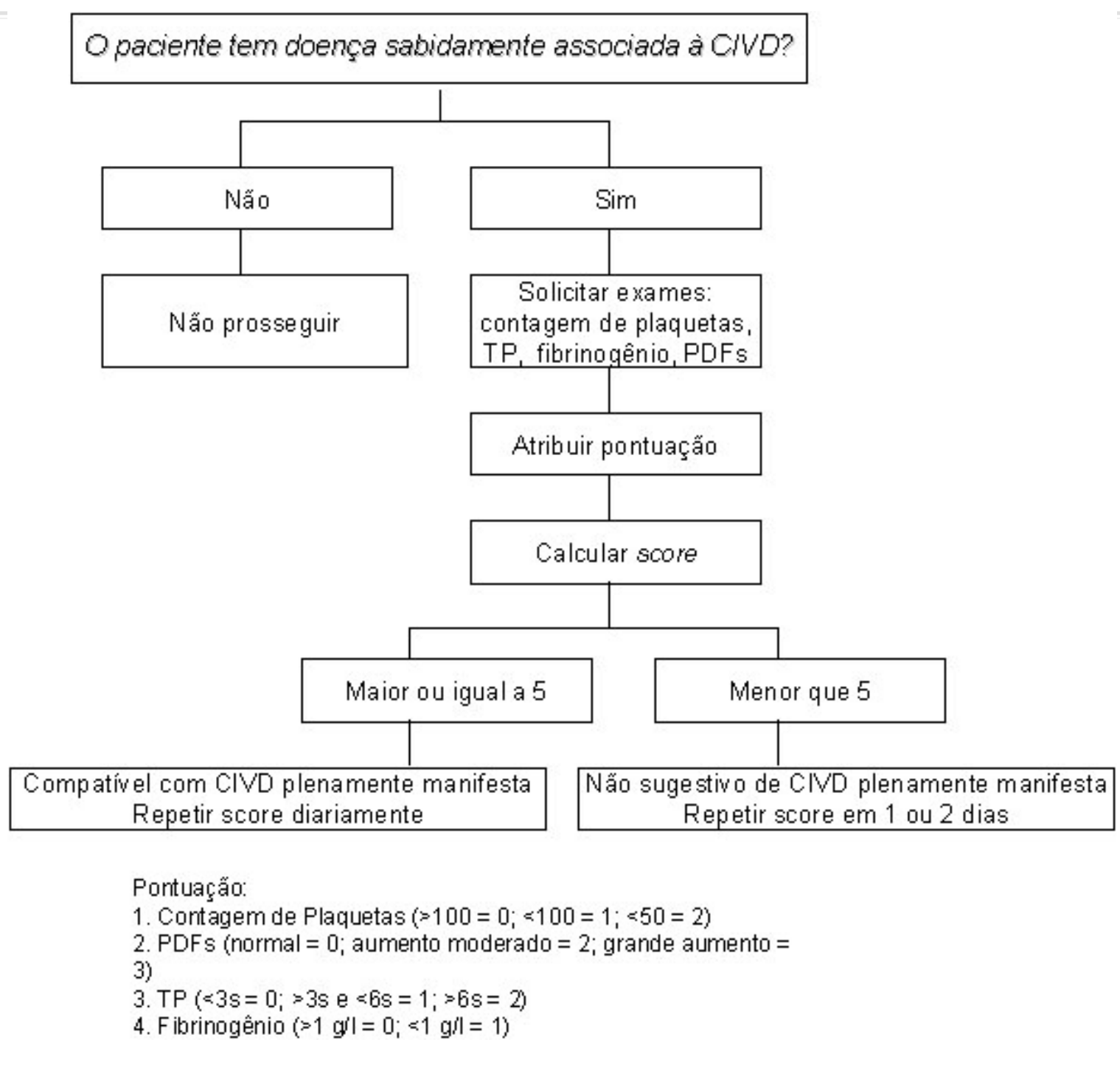

Figura 7: Algoritmo para diagnóstico de CIVD plenamente manifesta

se tem sobre a fisiopatologia da CIVD podem ser implementadas. De forma geral, as intervenções se baseiam no uso de anticoagulantes, reposição de plasma e plaquetas e administração de inibidores fisiológicos da coagulação, consoante detalhado a seguir (Figura 8$)^{(1,2)}$.

Anticoagulantes. Estudos experimentais mostraram que a heparina pode, pelo menos em parte, inibir a ativação da coagulação relacionada à septicemia e outras causas. Estudos não controlados, envolvendo pacientes com diagnóstico de CIVD sugeriram benefício do uso de heparina, porém tal benefício não foi, até o momento, inequivocamente demonstrado em estudos controlados e incluindo grande número de
- Tratamento da doença de base

- Medidas de "suporte"

- Anticoagulantes

Heparina, LMWH: dose

Inibidores de trombina

Anti-TF (rNAPc2)

- Transfusão de plaquetas

- Transfusão de plasma e crioprecipitado

- Concentrados de inibidores (AT, PC, TFPI)

- Antifibrinolíticos: contra-indicados

Figura 8: CIVD: tratamento 
pacientes. Além disto, a segurança do tratamento com heparina em pacientes com risco de sangramento também é motivo de debate. Por outro lado, a profilaxia com heparina pode prevenir tromboembolismo nos pacientes com CIVD. A administração de doses profiláticas de heparina por via subcutânea ou endovenosa ainda é recomendada em pacientes com CIVD em numerosos centros. Doses maiores devem ser reservadas para pacientes com tromboembolismo ou situações de deposição extensa de fibrina como purpura fulminatus e isquemia acral. Quando se toma a decisão de se utilizar a heparina, tanto a heparina não fracionada como a heparina de baixo peso molecular podem ser administradas.

O uso de inibidores da trombina não dependentes da AT, como a hirudina, pode ser benéfico em pacientes com CIVD, porém não existem ainda estudos clínicos envolvendo pacientes com CIVD que autorizem seu uso e o alto risco de sangramento pode ser um fator limitante importante para sua aplicação em tais pacientes.

Transfusão de plasma e plaquetas. Baixos níveis de fatores da coagulação e plaquetas podem aumentar o risco de sangramento na CIVD. No entanto, a transfusão desses produtos não deve ser feita apenas com base em exames laboratoriais, mas também, no quadro clínico do paciente. Não existem estudos bem controlados que provem a eficácia da transfusão "profilática" de plasma e plaquetas, mas ela parece ser uma opção racional para pacientes com sangramento e para aqueles que vão ser submetidos a procedimento invasivo. Reposição de concentrados de fatores da coagulação não está indicada.

Concentrado de inibidores da coagulação. A restauração das vias naturais de anticoagulação parece ser um objetivo terapêutico adequado. A PC é um anticoagulante natural que promove fibrinólise, inibe trombose e inflamação e é um importante modulador desses processos em pacientes sépticos. Bernard et al. demonstraram recentemente num estudo clínico multicêntrico, duplo cego e randomizado (PROWESS), que, em pacientes com septicemia grave e evidências de disfunção de múltiplos órgãos (choque circulatório, acidose, oligúria e hipoxemia), o uso da PC recombinante foi eficiente em diminuir a mortalidade, independente da idade, gravidade da doença, número de órgãos comprometidos, sítio da infecção ou tipo de organismo envolvido. $\mathrm{O}$ aumento de risco hemorrágico não atingiu significância estatística, porém deve-se ressaltar que o delineamento desse estudo excluiu pacientes com alto risco de sangramento (hepatopatia crônica, nefropatia crônica diálise-dependente, intervenção cirúrgica recente ou transplante, trombocitopenia [contagem de plaquetas $<30.000 / \mathrm{mm}^{3}$ ] e uso de ácido acetil salicílico [dose maior que $650 \mathrm{mg} / \mathrm{dia}$ ] até três dias antes do início do estudo). Outros estudos são, portanto, necessários para avaliar a eficácia do tratamento em pacientes com septicemia moderada e leve, sem disfunção de órgãos, e em pacientes sob maior risco hemorrágico. De qualquer forma, o PROWESS forneceu evidências clínicas claras de que novas terapias utilizando agentes com propriedades antiinflamatórias e anticoagulantes, são eficazes em diminuir a mortalidade em pacientes sépticos $\operatorname{graves}^{(9,15)}$.

A antitrombina também é um importante inibidor fisiológico da coagulação. Estudos em modelos animais e em humanos (envolvendo pequeno número de pacientes), mostram resultados promissores com o uso de concentrados de antitrombina, porém ainda não foram publicados estudos clínicos adequadamente delineados, que provem sua eficácia a ponto de autorizar seu uso na rotina terapêutica de pacientes com CIVD. Outras modalidades terapêuticas, como anticorpos anti-FT (rNAPc2), TFPI, trombomodulina recombinante e intervenções em nível de citocinas, encontram-se atualmente sob avaliação ${ }^{(2)}$.

PINTÃO MCT \& FRANCO RF. Disseminated intravascular coagulation. Medicina, Ribeirão Preto, 34: 282291, july/dec. 2001.

ABSTRACT: In the present article we review several aspects related to the etiology, pathophysiology, diagnosis and treatment of disseminated intravascular coagulation (DIC).

UNITERMS: Disseminated Intravascular Coagulation. Coagulation. Inflammation. 


\section{REFERÊNCIAS BIBLIOGRÁFICAS}

1 - LEVI M \& TEN CATE H. Disseminated intravascular coagulation. N Engl J Med 341: 586-592, 1999.

2 - LEVI M; DE JONGE E; VAN DER POLL T \& TEN CATE H. Disseminated intravascular coagulation. Thromb Haemost 82: 695-705, 1999.

3 - TAPPER H \& HERWALD H. Modulation of hemostatic mechanisms in bacterial infectious diseases. Blood 96: 2329-2337, 2000.

4 - TEN CATE H; TIMMERMAN JJ \& LEVI M. The pathophysiology of disseminated intravascular coagulation. Thromb Haemost 82: 713-717, 1999.

5 - FRANCO RF; DE JONGE E; DEKKERS PEP; TIMMERMAN JJ; SPEK CA; VAN DEVENTER SJH; VAN DEURSEN P; VAN KERKHOFF L; TEN CATE H; VAN DER POLL T \& REITSMAPH. The in vivo kinetics of tissue factor messenger RNA expression during human endotoxemia: relationship with activation of coagulation. Blood 96: 554-559, 2000.

6 - ESMON CT; FUKUDOME K; MATHER T; BODE W; REGAN LM; STEARNS-KUROSAWA DJ \& KUROSAWA S. Inflammation, sepsis and coagulation. Haematologica 84: 254-259, 1999.

7 - VAN GORP ECM; SUHARTI C; TEN CATE H; DOLMANS WM; VAN DER MEER JW; TEN CATE JW \& BRANDJES DP. Review: infectious diseases and coagulation disorders. J Infect Dis 180: 176-186, 1999.

8 - LEVI M; VAN DER POLL T; TEN CATE H \& VAN DEVENTER $\mathrm{SJH}$. The cytokine-mediated imbalance between coagulant and anticoagulant mechanisms in sepsis and endotoxaemia. Eur J Clin Invest 27: 3-9, 1997.
9 - BERNARD GR; VINCENT JL; LATERRE PF; LAROSA SP; DHAINAUT JF; LOPEZ-RODRIGUEZ A; STEINGRUB JS; GARBER GE; HELTERBRAND JD; ELY EW \& FISHER CJ JR. Efficacy and safety of recombinant human activated protein C for severe sepsis. N Engl J Med 344: 699-709, 2001.

10 - TEN CATE H; SCHOENMAKERS SHHF; FRANCO RF; TIMMERMAN JJ; GROOT AP; SPEK CA \& REITSMA PH. Microvascular coagulopathy and disseminated intravascular coagulation. Crit Care Med 29: S95-98, 2001.

11 - MARSHALL JC. Inflammation, coagulopathy, and the pathogenesis of multiple organ dysfunction syndrome. Crit Care Med 29: S99-106, 2001.

12 - BICK RL. Disseminated intravascular coagulation: objective criteria for clinical and laboratory diagnosis and assessment of therapeutic response. Semin Thromb Haemost 24:3-18, 1998.

13 - MULLER-BERGHAUS G; TEN CATE H \& LEVI M. Disseminated intravascular coagulation: clinical spectrum and established as well as new diagnostic approaches. Thromb Haemost 82: 706-712, 1999.

14 - TAYLOR JR FB; TOH CH; HOOTS WK; WADA H \& LEVI M. Towards definition, clinical and laboratory criteria, and a scoring system for disseminated intravascular coagulation. Thromb Haemost 86: 1327-1330, 2001.

15 - MATTHAY MA. Severe sepsis - a new treatment with both anticoagulant and anti-inflammatory properties. N Engl J Med 344: 759-762, 2001.

Recebido para publicação em 28/06/2001

Aprovado para publicação em 24/08/2001 